\title{
A entrada na Europa e a expansão inicial do eucalipto em Portugal Continental
}

\author{
João Paulo S. Cabral
}

\section{Resumo}

As primeiras observações e recolhas de eucaliptos ocorreram nas grandes viagens inglesas e francesas ao Pacífico, em particular à Austrália, em finais do século XVIII. O género Eucalyptus L'Hér. foi estabelecido em 1788, e logo nas duas décadas seguintes seriam descritas, por botânicos franceses e ingleses, muitas espécies novas. O primeiro eucalipto cultivado em Inglaterra foi trazido, em 1774, na segunda viagem de James Cook. Em França, a introdução terá sido feita em 1804, no Jardim Botânico de Montpellier, na Alemanha em 1809, no Jardim Botânico de Berlim, e em Itália, em 1813, no Jardim Botânico de Nápoles. Em Portugal, a introdução do eucalipto foi muito posterior a estas datas. Na propriedade do duque de Palmela no Lumiar, foram plantados dois eucaliptos em 1850-1852. No Horto Botânico da Escola Médico-Cirúrgica de Lisboa, existia em 1852, pelo menos um espécimen, certamente para uso ou demonstração das suas propriedades terapêuticas. A partir da década de 1860, a expansão foi muito rápida. Em 1869, a companhia real dos caminhos-de-ferro portugueses iniciou a plantação de eucaliptos nas estações, casas de guarda e ao longo da via-férrea. As primeiras plantações em larga escala terão ocorrido na década de $1880 \mathrm{em}$ propriedades perto de Abrantes arrendadas por William T. Tait. Em 1886 estavam já plantados 150 mil eucaliptos. Nesta mesma década começou a plantação, em escala apreciável, de eucaliptos nas Matas Nacionais. Em finais do século XX, tinham sido introduzidas em Portugal cerca de 250 espécies, sendo o Eucalyptus globulus Labill., a espécie largamente dominante. É interessante constatar que tendo sido um dos países europeus que mais tarde introduziu a cultura do eucalipto, Portugal é hoje, a nível mundial, um dos que apresenta maior percentagem da sua área florestal dedicada a esta cultura.

Palavras-chave: eucalipto; jardins botânicos; Portugal.

\begin{abstract}
The earliest observations and collections of eucalypts occurred on the great English and French voyages to the Pacific, particularly Australia, in the late 18th century. The genus Eucalyptus L'Hér. was described in 1788, and soon in the following two decades, many species would be described by French and English botanists. The first eucalypt grown in England was brought in 1774 on James Cook's second voyage. In France, the introduction seems to have occurred in 1804, at the Botanical Garden of Montpellier, in Germany in 1809, at the Botanical Garden of Berlin, and in Italy, in 1813, at the Botanical Garden of Naples. In Portugal, the introduction of eucalypts was much later than these dates. In the property of the Duke of Palmela in Lumiar, two eucalypts were planted in 1850-1852. The Botanical Garden of the Medical-Surgical School of Lisbon had in 1852, at least one specimen, certainly for use or demonstration of its therapeutic properties. From the 1860s the expansion was very rapid. In 1869, the royal company of the Portuguese railways began planting eucalypts in the stations, guard houses and along the railroad. The first large-scale plantations occurred in the 1880s in properties near Abrantes leased by William T. Tait. By 1886, 150,000 eucalypts were already planted. In the same decade began the planting, on an appreciable scale, of eucalypts in "Matas Nacionais". By the end of the 20th century about 250 species had been introduced in Portugal, being Eucalyptus globulus Labill., the species largely dominant. It is interesting to note that Portugal, one of the European countries that later introduced the eucalypt, is today, worldwide, one of the countries with the highest percentage of its forest area dedicated to this culture.
\end{abstract}

Keywords: eucalypt; botanical gardens; Portugal. 


\section{A EXPLORAÇÃO dO PACíFICO E DO CONTINENTE AUSTRALIANO}

Em finais do século XVIII, o domínio do Pacífico estava na mira das três principais potências marítimas - Inglaterra, França e Espanha. Buscavam-se novos pontos de aprovisionamento para os navios que atravessavam este oceano e, se possível, novas bases para 0 estabelecimento de intercâmbio ou domínio comercial. A gigantesca dimensão deste oceano ${ }^{1}$, o medo dos navegantes em penetrar nas águas calmas do seu interior, a terrível ameaça do escorbuto que dizimava tripulações inteiras, a imprecisão na determinação da longitude (que podia atingir $5-10^{\circ}$, equivalentes a $500-1.000$ quilómetros), levavam a uma pervivência das rotas tradicionais e ao medo em empreender novas aventuras ${ }^{2}$.

As três grandes viagens comandadas por James Cook (1728-1779) direcionadas para 0 reconhecimento do Pacífico, despertaram um enorme interesse na comunidade científica e nas potências europeias marítimas. Na primeira viagem, iniciada em 1768, Cook cartografou as costas da Nova Zelândia, atravessou o Mar da Tasmânia e a Grande Barreira de Coral, chegando à costa sudeste da Austrália a 19 de Abril de 1770. Joseph Banks (1743-1820) e Daniel Solander (1733-1782) recolheram plantas na Botany Bay, na costa leste australiana. Na segunda missão, entre 1772 e 1775, Cook descobriu e localizou várias ilhas do Pacífico e no Atlântico Sul. Johann Reinhold Forster (17291798), J. George A. Forster (1754-1794) e Anders Sparrman (1748-1820) recolheram plantas na Adventure Bay (Tasmânia). Na terceira viagem, que partiu de Inglaterra em 1776, Cook confirmou que não existe uma passagem viável entre o Pacífico e o Atlântico, à volta do Canadá e do Alasca, ou à volta da Sibéria. David Nelson (?-1789) e William Anderson (1750-1778) recolheram plantas na Tasmânia ${ }^{3}$. Os materiais recolhidos durante as três viagens de Cook foram distribuídos entre académicos e instituições, sob a coordenação de J. Banks. J. R. Forster enviou alguns materiais para Oxford, A. Sparrman para a Academia Sueca de Ciências, James Patten ${ }^{4}$, para o Trinity College de Dublin, e John Webber (1751-1793) para o Museu de Berna5.

\footnotetext{
${ }^{1}$ Se imaginarmos um triângulo cujos vértices estão na Austrália, na Ilha de Páscoa e nas ilhas Havai, o seu lado teria cerca de 7 mil quilómetros (A. Lafuente, "Las expediciones cientificas del Setecientos y la nueva relacion del cientifico con el Estado" Revista de Indias, 47 (1987): 376.

2 Ibid.

3 P. Grimal, dir., Dictionnaire des Biographies, vols. 1 e 2 (Paris: Presses Universitaires de Prance, 1958), 364-365; E. C. Nelson, "The locations of collection and collectors of specimens described by La Billardière in Novae Hollandiae Plantarum Specimen. Additional notes." Papers and Proceedings of the Royal Society of Tasmania 108 (1974): 159-170; Jacques Brosse, Great Voyages of Discovery. Circumnavigators and scientists, 1764-1843 (New York: Facts On File Publications, 1983), 63-74.

${ }^{4}$ Em 1772 era cirurgião a bordo do navio Resolution.

${ }^{5}$ C. E. Harrison, "Projections of the revolutionary nation: French expeditions in the Pacific, 17911803" Osiris 24 (2009): 33-52.
} 
Além da Inglaterra, também a França levou a cabo grandes expedições destinadas a explorar o oceano Pacífico6 ${ }^{6}$. A primeira partiu em 1785, comandada por Jean-François de Galaup de La Pérouse (1741-1788) e terminará tragicamente em 1788 nas ilhas Salomão com o naufrágio dos dois navios $^{7}$. O facto de os navios não terem regressado a França dentro do prazo previsto, nem se conhecer o seu paradeiro, levou à organização de uma nova expedição. Esta missão foi chefiada por Antoine Reymond Bruny d'Entrecasteaux (1737-1793), tendo partido de França em 1791. Comandava duas fragatas que exploraram os rios da Nova-Zelândia, a Nova-Caledónia, as ilhas Tonga e as costas da Austrália e da Tasmânia. A bordo da fragata La Recherche seguiu o botânico Jacques-Julien Houtou de La Billardière (1755-1834). Seria o primeiro francês a chegar à Austrália e à Tasmânia e a descrever a sua história natural. Terá também um desfecho trágico, tendo d'Entrecasteaux morrido em julho de 1793 no oceano Pacífico, entre a Tasmânia e Java. Parte da tripulação foi depois capturada pelos Holandeses em Java e, de seguida, presa pelos Ingleses. Os materiais de história natural foram confiscados e enviados para Inglaterra, tendo, no entanto, La Billardière conservado os seus manuscritos de viagem. Regressado a França em 1796, La Billardière pediu a Joseph Banks a devolução das suas coleções, em particular o seu herbário com cerca de quatro milhares de plantas, 0 que se concretizou 8 .

A terceira expedição francesa ao Pacífico decorreu entre 1800 e 1804, e foi comandada por Nicolas Thomas Baudin (1754-1803). Foi explorada a Geographe Bay e a Shark Bay, na Austrália, tendo depois rumado a Timor. Regressou à Austrália ocidental, recolhendo material em King George Sound. Uma parte da coleção de história natural recolhida em Geographe Bay e Shark Bay foi logo enviada para França em 1803 a bordo da fragata Le Naturaliste. A segunda embarcação - Le Géographe, regressou em 1804 e trouxe os restantes materiais de história natural, nomeadamente a coleção recolhida em King George Sound. A viagem foi também trágica e atribulada. N. T. Baudin morreu na viagem de regresso, na ilha Maurícia. J.-B. Leschenault de la Tour (1773-1826) adoeceu e desembarcou em Koepang (Timor ocidental), só regressando a França em 1807. De um total de 16 académicos que seguiram na viagem, só cinco regressaram a França, sendo Guichenot, jardineiro horticultor, o único botânico a regressar. $O$ relato da viagem, incluindo as cartas, foi redigido por Péron e depois da sua morte, por Freycinet?

6 Ao contrário das expedições inglesas, nas quais os interesses científicos iam a par dos geoestratégicos e comerciais, as expedições francesas eram patrocinadas pela coroa e os objetivos eram prioritariamente de índole científica. Todos os resultados das expedições francesas eram propriedade do Estado. As instituições parisienses eram o primeiro destino dos objetos trazidos de além-mar, ao contrário do que ocorria com as viagens inglesas, nas quais os exemplares eram distribuídos por académicos e instituições, muitas das quais eram estrangeiras (Ibid.).

7 Brosse, Great Voyages of Discovery, 75-83.

8 Harrison, Projections of the revolutionary nation.

9 Ibid. 


\section{PRIMEIRAS OBSERVAÇÕES E ESTUDOS DOS EUCALIPTOS}

Foi numa ilha da Espérance Bay, na costa sudoeste da Austrália que La Billardière observou, estudou e recolheu espécimenes de várias espécies de eucaliptos ${ }^{10}$. La Billardière apresentaria os seus resultados de taxonomia botânica nas obras Relation du Voyage ${ }^{11}$ e Novae Hollandiae Plantarum Specimen ${ }^{12}$. Descreveria 265 plantas australianas, da Nova Holanda, estando 77 indicadas como tendo sido recolhidas na Austrália ocidental - "Terra van-Leuwin", sendo 54 endémicas desta região13. Para este trabalho de taxonomia botânica, recorreu a material de história natural da sua expedição bem como da expedição seguinte, de N. T. Baudin ${ }^{14}$.

Antes de La Billardière, já Charles Louis L'Héritier de Brutelle (1746-1800), na obra Sertum Anglicum ${ }^{15}$, tinha estabelecido o género Eucalyptus L'Hér. e a espécie Eucalyptus obliqua L'Hér. Logo depois, outros botânicos de várias nacionalidades, como James Edward Smith (1759-1828), A. J. Cavanilles (1745-1804) e James Donn (1758-1813), descreveram mais espécies novas de eucaliptos ${ }^{16}$.

\section{OS PRIMEIROS EUCALIPTOS CULTIVADOS EM JARDINS BOTÂNICOS EUROPEUS}

10 Conhecem-se atualmente largas centenas de espécies do género Eucalyptus L'Hér., quase todas endémicas da Austrália e Tasmânia, com exceção de seis, das quais se salientam, Eucalyptus alba Reinw. ex Blume que ocorre também nas ilhas de Sunda e Nova Guiné e Eucalyptus urophylla S.T. Blake que ocorre nas ilhas de Sunda. Somente cerca de uma dezena de espécies tiveram grande expansão fora da sua área de distribuição nativa. Destas destaca-se o Eucalyptus globulus Labill., a espécie mais importante nos climas temperados e o Eucalyptus camaldulensis Dehnh., a espécie com maior expansão a nível mundial. Na Austrália e na Tasmânia, cerca de $80 \%$ das florestas naturais são constituídas por eucaliptos. Estas formações naturais apresentam uma ou várias espécies de eucaliptos, com um sub-bosque normalmente de acácias. O crescimento dos eucaliptos na Austrália é cerca de 3-4 vezes inferior ao que se observa nos países onde foi introduzido, em consequência das muitas pragas e doenças que o afetam na sua área de distribuição nativa ( $E$. Goes, Os Eucaliptos (Ecologia, cultura, produções e rentabilidade) (s.l.: Portucel, 1977)).

11 J. J. H. de La Billardière, Relation du voyage à la recherche de La Pérouse, vols. 1, 2 e Atlas (Paris: H. J. Jansen, 1800).

12 J. J. H. de La Billardière, Novae Hollandiae plantarum specimen, vols. 1 e 2 (Parisiis: Huzard, 1804-1806).

${ }^{13}$ As espécies novas de Eucalyptus observadas na Austrália, descritas por La Billardière no volume I da Relation du Voyage e no volume II da sua obra Novae Hollandiae Plantarum Specimen, apresentando desenhos ilustrativos, foram as seguintes: Eucalyptus amygdalina Labill.; Eucalypus cordata Labill.; Eucalyptus cornuta Labill.; Eucalyptus globulus Labill.; Eucalyptus incrassata Labill.; Eucalyptus ovata Labill.; Eucalyptus viminalis Labill. La Billardière faz referência a uma espécie já existente: Eucalyptus resinifera Sm., descrita poucos anos antes descrita por James Edward Smith na sua obra Journal of a Voyage to New South Wales, publicada em 1790.

14 A. Chevalier, "Un grand voyageur naturaliste normand: J.-J. La Billardière (1755-1834)" Revue internationale de botanique appliquée et d'agriculture tropicale, 33 (1953): 97-124; E. C. Nelson, "The Collectors and Type Locations of Some of Labillardière's "terra van-Leuwin" (Western Australia) Specimens." Taxon 24 (1975): 319-336; S. G. M. Carr \&. D. J. Carr, "La contribution de la France à la découverte de l'Australie et de sa flora." Endeavour, Edition française, 35 (1976): 21-26.

15 Neste trabalho publicado em 1788, L'Héritier descreve plantas raras que existiam em jardins da região de Londres.

16 Eucalyptus botryoides Sm.; Eucalyptus capitellata Sm.; Eucalyptus corymbosa Sm.; Eucalyptus haemastoma Sm.; Eucalyptus paniculata Sm.; Eucalyptus pilularis Sm.; Eucalyptus piperita Sm.; Eucalyptus robusta Sm.; Eucalyptus saligna Sm.; Eucalyptus tereticornis Sm.; Eucalyptus rostrata Cav.; Eucalyptus marginata Donn ex Sm. 
Cerca de duas décadas de exploração do continente australiano tinham resultado na observação, estudo, caracterização e descrição de várias dezenas de espécies de eucaliptos. Em inícios do século XIX, os eucaliptos não eram, de forma alguma, plantas desconhecidas, de um ponto de vista de taxonomia botânica. Quanto tempo demorou para que eucaliptos fossem trazidos para a Europa e cultivados em parques privados e em jardins botânicos? Muito pouco tempo!17 O primeiro eucalipto cultivado em Inglaterra foi trazido em 1774 pelo capitão Tobias Furneaux (1735-1781), que comandou a fragata Adventure, na segunda viagem de J. Cook ${ }^{18}$. Em princípios do século XIX, o eucalipto já crescia em vários jardins botânicos europeus. Em França, a introdução parece ter sido feita em 1804, no Jardim Botânico de Montpellier ${ }^{19}$, na Alemanha em 1809, no Jardim Botânico de Berlim²0, em Itália, em 1813, no Jardim Botânico de Nápoles²1 e no Jardim Botânico de Monza²2, e na Holanda em 1818 no Jardim Botânico de Leiden ${ }^{23}$. Em alguns jardins botânicos, nomeadamente no de Paris, sob a direção de Desfontaines, e no de Berlim com a direção de J. F. Link, faz-se investigação sobre a taxonomia deste género, com a descrição de espécies novas.

O Jardim Camaldulensis de Nápoles foi criado em 1816 por Francesco Ricciardi, conde de Camaldoli. O primeiro catálogo do jardim foi publicado em 1829, por Friedrich Dehnhardt. Na extensa e excecional lista de plantas em cultura são mencionadas seis espécies de eucaliptos, das quais uma era uma espécie nova - Eucalyptus elata Dehnh. ${ }^{24}$. Três anos depois, o número de espécies e variedades de Eucalyptus L'Hér. em cultura no jardim era já de doze, das quais quatro espécies e uma variedade eram novas: Eucalyptus camaldulensis Dehnh.; Eucalyptus gigantea Dehnh.; Eucalyptus linearis Dehnh.; Eucalyptus procera Dehnh.; Eucalyptus pulverulenta var. ovatifolia Dehnh. ${ }^{25}$. Neste catálogo de 1832 era apresentado um mapa topográfico do jardim com indicação da localização das "Das plantas mais raras de climas mais quentes" aclimatadas "no jardim camaldulense, distribuídas em famílias ou

\footnotetext{
17 Através da consulta das listas de plantas em cultura em parques privados e jardins botânicos é possível seguir, com assinalável pormenor, a cronologia da entrada do eucalipto na Europa, da qual apresentamos no presente trabalho, um resumo. Trata-se de um tema interessante para ser abordado no âmbito do ensino avançado da História da Botânica.

18 R. A. Salisbury \& W. Hooker, The Paradisus Londinensis: containing plants cultivated in the vicinity of the metropolis, vol. I, part I (London: D. N. Shury, 1806); W. T. Aiton, Hortus Kewensis, or a Catalogue of the Plants Cultivated in the Royal Botanic Garden at Kew, 2. ${ }^{a}$ ed., vol. 3 (London: Longman, Hurst, Rees, Orme, and Brown, 1811), 192-194.

19 P.-M.-A. Broussonet, Elenchus Plantarum Horti Botanici Monspeliensis. Anno 1804 (Monspelii: Ex. Officina Augusti Ricard, 1805).

20 C. L. Willdenow, Enumeratio Plantarum Horti Regii Botanici Berolinensis (Berolini: In Taberna Libraria Scholae Realis, 1809), 515.

21 M. Tenore, Ad Catalogum Plantarum Horti Regii Neapolitani Anno 1813 editum. Appendix Prima (Neapoli: Ex Typographia Amuliana, 1815), 8.

22 Catalogus plantarum existentium in Hortis Regiae Villae prope Modoetiam (Modoetiae: Typis L. Corbetta, 1813), 29.

23 S. J. Brugmans, Elenchus Plantarum quae in Horto Lugdano-Batavo coluntur (s.I., s.ed., 1818, 1819).

${ }^{24}$ F. Dehnhardt, Catalogus Plantarum Horti Camaldulensis (s.l., s.ed., 1829).

25 F. Dehnhardt, Catalogus Plantarum Horti Camaldulensis 2.a ed. (s.I., s.ed., 1832).
} 
colocadas isoladamente de acordo com a necessidade de sua posição mais ou menos quente"26. Os eucaliptos encontravam-se essencialmente distribuídos em duas zonas do jardim. É também dada indicação da idade, altura e diâmetro dos eucaliptos. A idade das árvores variava entre 15 e 5 anos. Tendo o jardim sido criado em 1816, existiam portanto eucaliptos que foram plantados praticamente logo no início deste estabelecimento. As espécies e a variedade nova tinham sido plantadas nos seguintes anos: Eucalyptus camaldulensis Dehnh. - 1822; Eucalyptus elata Dehnh. - 1817; Eucalyptus gigantea Dehnh. - 1826; Eucalyptus linearis Dehnh. - 1827; Eucalyptus procera Dehnh. - 1827; Eucalyptus pulverulenta var. ovatifolia Dehnh. - 1824.

\section{ENTRADA E PROGRESSÃo INICIAL DOS EUCALIPTOS EM PORTUGAL}

Em Portugal a introdução do eucalipto foi tardia, muito posterior à que ocorreu em Inglaterra, França e Itália. Em 1828, quando morreu, F. A. Brotero deixou uma lista das plantas em cultura no Jardim Botânico da Ajuda, em Lisboa, não sendo mencionada qualquer espécie de eucalipto ${ }^{27}$. Pedro José de Noronha de Albuquerque Moniz e Sousa (1716-1788), 3. ${ }^{\circ}$ marquês de Angeja, tinha na sua grande propriedade ao Lumiar $^{28}$, uma coleção de plantas exóticas e notáveis, à qual Domingos Vandelli terá dado orientação e apoio ${ }^{29}$. Estando já a propriedade na posse do duque de Palmela, foram plantados, em 1850-1852, dois eucaliptos ${ }^{30}$ que, em 1858, apresentavam mais de 10 metros de altura e quase um metro de circunferência do tronco ${ }^{31}$. Em 1877-1880, António Ricardo da Cunha, colector de plantas da Escola Politécnica de Lisboa, preparou folhas de herbário de 97 espécies de plantas lenhosas ${ }^{32}$. Permanece material de herbário de três espécies de eucaliptos: Eucalyptus diversicolor F. Muell., Eucalyptus robusta Sm. e Eucalyptus stuartiana F. Muell. ex Miq. Quando em 1877 foram recolhidas as amostras, eram árvores relativamente jovens, tendo 7, 18 e 4 metros de altura e 0,16,

\footnotetext{
${ }^{26}$ Ibid., 25.

27 F. A. Brotero, "Historia Natural. Botanica. Continuação do Catalogo Geral de todas as Plantas do Nacional e Real Jardim Botanico d'Ajuda." Jornal da Sociedade Pharmaceutica de Lisboa 1 (18361837): 445-452.

28 Esta propriedade constituiu-se por compras sucessivas a vários proprietários. Foi transmitida dentro da casa Angeja até 1840 quando foi vendida a Domingos de Sousa Holstein Beck (18181864), $1 .^{\circ}$ marquês de Faial e $2 .^{\circ}$ duque de Palmela. Permaneceu na família Palmela até 1976 quando foi comprada pelo Estado Português para instalar o Museu do Teatro e do Traje, que continuam a funcionar (B. A. Alves, "Plantas florestaes e de ornamento naturalisadas na Quinta do Lumiar." O Archivo Rural, Jornal de Agricultura Artes e Sciencias Correlativas 1. ${ }^{\circ}$ anno (1858): 323-325).

29 L. F. C. F. A. Lara, Parque do Monteiro-Mor. Inventário das árvores, arbustos e plantas herbáceas do Parque do Monteiro-Mor (Lisboa: Museu Nacional do Traje, 1987).

30 Eucalyptus globulus Labill. e possivelmente de Eucalyptus falcata Turcz (Alves, Plantas florestaes, 324)

${ }^{31}$ Ibid., 324.

32 Herbário atualmente no Instituto Superior de Agronomia (Lisboa).
} 
0,40 e 0,12 metros de diâmetro, respetivamente ${ }^{33}$. Teriam sido plantadas depois dos dois espécimenes colocados na terra em 1850-1852.

Também em Lisboa, no Horto Botânico da Escola Médico-Cirúrgica, existia em 1852 pelo menos um espécimen de Eucalyptus robusta Sm. ${ }^{34}$, certamente para uso ou demonstração das suas propriedades terapêuticas. Num trabalho dedicado a espécies florestais exóticas com interesse económico para o nosso país, Edmond Goeze (1871) referia a utilidade das folhas e da casca de eucalipto para "tractar seriamente as febres intermittentes e mesmo a gota", e ainda "para o fabrico do papel", apresentando uma lista das espécies mais recomendada para esta última finalidade. As palavras seguintes de E. Goeze relevam claramente que o eucalipto era uma novidade em Portugal: "Ha pouco o Jardim Botânico de Coimbra recebeu do sábio director do estabelecimento botânico de Melbourne uma porção de semente do Eucalyptus marginata, o celebre "Mahagony" da Austrália, e em verdade, pela sua madeira preciosa, que resiste a toda a influencia da agua do mar, esta espécie nào tem rival entre as suas congéneres"35. Em 1873, E. Goeze referia que o Jardim Botânico de Coimbra tinha recebido do Jardim Botânico de Melbourne sementes de duas espécies de eucaliptos "inteiramente desconhecidas n'este paiz": Eucalyptus macrocarpa Hook e Eucalyptus citriodora Hook, plantas que são descritas por Goeze neste trabalho36. Terminava referindo que já tinha distribuído "indivíduos d'estas duas espécies a alguns amadores", e esperava "que as primeiras experiencias tenham bom resultado para assim enriquecer a nossa Flora d'arvores exoticas"37.

Estes vários elementos informativos corroboram a conclusão apresentada por A. X. Pereira Coutinho, no seu Curso de Silvicultura publicado em 1886-1887, que aponta a introdução do eucalipto em Portugal na década de 1850, estando então sobretudo restringido ao Eucalyptus globulus Labill. ${ }^{38}$

Depois da entrada inicial, como progrediu a cultura do eucalipto em Portugal durante o século XIX? Rapidamente! Carlos Augusto de Sousa Pimentel (?-1912), silvicultor dos serviços florestais, e Duarte de Oliveira Júnior (1848-1927) foram dos mais activos divulgadores e promotores da cultura do Eucalyptus globulus Labill. em Portugal. Várias características desta planta justificavam o interesse e a rapidíssima expansão: o crescimento "excepcionalmente" rápido; vegetam em terrenos "improductivos"; a madeira "é muito rija, resistente, e offerece grande duração, podendo ser usada na construção civil, de obras publicas e náutica; a casca pode ser usada na indústria dos curtumes; das folhas extraem-se

\footnotetext{
33 J. A. Franco \& M. L. M. R. Afonso, "Relação das árvores e arbustos mais notáveis do Parque do Monteiro-Mor" in Parque do Monteiro-Mor. Inventário das árvores, arbustos e plantas herbáceas do Parque do Monteiro-Mor (Lisboa: Museu Nacional do Traje, 1987) 30.

34 B. A. Gomes \& C. M. F. S. Beirão, Catalogus Plantarum Horti Botanici Medico-Cirurgicae Scholae Olisiponensis. Anno MDCCCLII (Olisipone: Typographia Nationali, 1852), 58.

35 E. Goeze, "Excursão botanica e horticola." Jornal de Horticultura Prática 2 (1871): 144-147.

36 E. Goeze, "Duas novas especies de Eucalyptus." Jornal de Horticultura Prática 4 (1873): 29-30.

37 Ibid.

38 A. X. Pereira Coutinho, Curso de silvicultura, vols. 1 e 2 (Lisboa: Typographia da Academia Real das Sciencias, 1886 e 1887), 404 e 161, respetivamente.
} 
produtos de aplicação medicinal; podem ser usados para secar zonas alagadas e pantanosas ${ }^{39}$. C. A. de Sousa Pimentel publica, em 1876, uma monografia de divulgação que é premiada na Exposição Hortícola Internacional do Porto de 1877. Em 1884 publica um trabalho mais aprofundado destinado a divulgar a cultura do eucalipto no país ${ }^{40}$. Por seu lado, Oliveira Júnior publica em 1870, na Typographia Lusitana do Porto, um folheto de divulgação - Breve noticia sobre o Eucalyptus globulus e a utilidade da sua cultura.

As primeiras plantações de eucaliptos começaram por volta de 1860 com árvores para ornamentação, tendo sido iniciada, no final desta década, a plantação de pequenas matas ${ }^{41}$. $\mathrm{Na}$ década de 1870, existiam já plantações com milhares de árvores em vários locais, como a quinta da Foja, próxima de Montemor-o-Velho, no choupal e na mata de Vale de Canas, na região da Figueira da Foz, no Monte das Flores, perto de Évora, propriedade de José Maria Eugénio de Almeida, na mata nacional de Valverde, no concelho de Alcácer do Sal42. Em 1869, a companhia real dos caminhos-deferro portugueses iniciou a plantação de eucaliptos nas estações, casas de guarda e ao longo da viaférrea. Em 1876, eram já mais de 40 mil as árvores plantadas por esta empresa ${ }^{43}$. No concelho de Alenquer, na Serra Galega, Graciano Franco Monteiro, iniciou, em 1871, a plantação de eucaliptos que, alguns anos depois, já atingiam as 20 mil árvores ${ }^{44}$. 0 barão de Viamonte, numa propriedade na região de Leiria tinha, na década de 1870, os exemplares mais altos do país - com 14 anos tinham atingido 30-36 metros de altura!45. Na década de 1880, o eucalipto começava a ter um "logar importante na silvicultura portugueza"46.

As vantagens desta cultura era tais que William C. Tait, numa propriedade de 200 hectares "de terreno inculto" perto de Abrantes (freguesia da Bemposta), que sugestivamente designa de "Nova Tasmania", arrendada em 1880, decide mandar plantar largos milhares de eucaliptos. Em 1886, estavam já plantadas 150 mil árvores ${ }^{47}$, e constituía, segundo Tait "a maior [plantação] que existe em Portugal, e provavelmente na Europa"48. Eucalyptus globulus Labill. era a espécie dominante, mas tinham também sido plantadas outras dezenas de espécies deste género: "Há tambem muitos Eucalyptus rostrata (Gommeiro vermelho), resinifera, colossea, marginata, sideroxylon, polyanthemos,

${ }^{39}$ S. Pimentel, Eucalypto globulus. Descripção, Cultura e Aproveitamento d'esta arvore. 2. ${ }^{\text {a }}$ ed. (Lisboa: Typographia Universal, 1884).

40 Ibid.

${ }^{41}$ Ibid., 12.

42 Ibid., $13-14$.

43 Ibid., 15.

44 Ibid., 16.

45 Ibid., 17.

46 Ibid., 12.

47 D. Oliveira Júnior, "Eucalyptus globulus." Jornal de Horticultura Prática 17 (1886): 110-112.

${ }^{48}$ W. Tait, "Cultura de acacias." Jornal de Horticultura Pratica 16 (1885): 246-248. 
bicolor, amygadalina e cerca de quarenta outras espécies de Eucalyptus"49. Satisfeito com a solução, Tait, numa carta dirigida ao abade de Miragaia, Pedro Augusto Ferreira, datada de 9 de Março de 1886, contrapunha a nova floresta à esterilidade do passado: "Ali só havia matto, ao qual se lançava o fogo de vez em quando, para com as cinzas estimular a nascença da pouca herva que servia de pasto a algumas cabras" 50 . Conforme refere nesta carta dirigida ao abade de Miragaia - Pedro Augusto Ferreira, datada de 9 de Março de 1886, W. Tait tinha em mente a produção de madeira: "O que levou a escolher esta localidade, foi não só a sua posição vantajosa para a conducção das madeiras, por ser perto da margem do rio Tejo e do caminho-de-ferro para Portugal e Hespanha"51. O crescimento rapidíssimo dos eucaliptos não escapou à observação de W. Tait que, ainda nesta carta, o quantificava: "um Eucalyptus globulus plantado em Outubro de 1880 - media [quando escreve a carta seis anos depois] 52 centimetros de circumferencia a 1 metro do solo, e teria approximadamente 12 metros de altura"52.

Em 1886, na mesma revista e na sequência do artigo de Tait, Duarte de Oliveira Júnior transcrevia as cartas trocadas entre Pedro Augusto Ferreira, abade de Miragaia, no Porto, e W. C. Tait. Este clérigo tinha um elevado apreço por esta novel espécie florestal, sugerindo que fosse promovida a sua cultura pelos "grandes proprietarios d'esta província (Beira Baixa) e da do Alemtejo, nomeadamente [pelos] donos das grandes herdades nuas e de charneca medonha". Pedro Augusto Ferreira já se teria apercebido da capacidade do eucalipto em retirar água do solo dado que o indicava em especial para "terrenos em que predominam as febres intermittentes ${ }^{53}$, como são os campos de Coimbra, Leiria e Riba-Tejo, e o Alto Douro, nomeadamente a Barca d'Alva, medonho foco de sezões". "Quanto não lucraria a hygiene, se os nossos arrozaes fossem cultivados de Eucalyptus?".

As primeiras plantações de eucaliptos em muito grande escala foram feitas em 1880-1885, por W. C. Tait perto de Abrantes, como já foi referido. Nesta época eram as maiores do país. Uma das propriedades foi plantada principalmente com Eucalyptus globulus Labill., com sub-bosque de acácias (Acacia mollissima Willd., Acacia pycnantha Benth. e Acacia dealbata Link), tendo a outra sido arborizada essencialmente com Eucalyptus globulus Labill. e Eucalyptus camaldulensis Dehnh. Em ambas existia uma coleção diversificada de espécies ${ }^{54}$.

As plantações de eucaliptos nas Matas Nacionais começaram aproximadamente ao mesmo tempo que nas propriedades privadas. Nos pinhais nacionais de Leiria e da Foja começaram a ser plantados eucaliptos em 1879, por iniciativa de João Maria de Magalhães, tendo depois sido

\footnotetext{
49 Oliveira Júnior, Eucalyptus globulus, 111.

50 Ibid., 111.

51 Ibid., 111.

52 Ibid., 111.

53 Duarte de Oliveira Júnior estaria a referir-se ao paludismo.

54 Goes, Os Eucaliptos, 25-26.
} 
continuados por Sousa Pimentel. O Eucalyptus globulus Labill. começou a ser plantado em escala apreciável em 1883, nas Matas de Valverde, Gaio e Leiria. Em 1906, os serviços florestais plantaram na Mata Nacional das Virtudes, perto de Azambuja, um arboreto com mais de meia centena de espécies. As plantações na Mata Nacional do Escaroupim, perto de Muge, e na Mata Nacional do Urso, na região da Figueira da Foz, começaram em $1910^{55}$.

Em finais do século XX tinham sido introduzidas em Portugal cerca de 250 espécies, sendo Eucalyptus globulus Labill., a espécie largamente dominante, seguido de Eucalyptus camaldulensis Dehnh. (Baixo Alentejo) e Eucalyptus maidenii F. Muell. (Alentejo). As restantes espécies estavam restringidas a jardins, parques, povoamentos experimentais, arboretos ${ }^{56}$. Segundo Ernesto Goes, em 1977, a Mata Nacional de Escaroupim tinha cerca de 125 espécies, a Mata Nacional das Virtudes, mais de 60, as propriedades que foram de W. C. Tait perto de Abrantes, cerca de 40, a Quinta de São Francisco no Eixo, próxima de Aveiro, cerca de 7057.

Como comentário final, não deixa de ser interessante constatar que tendo sido um dos países europeus que mais tarde introduziu a cultura do eucalipto, Portugal seja hoje, a nível mundial, um dos que apresenta maior percentagem da sua área florestal com esta cultura58!

\section{SOBRE OS AUTORES:}

João Paulo S. Cabral

Faculdade de Ciências da Universidade do Porto (Portugal)

jpcabral@fc.up.pt; jpscabral@hotmail.com

\footnotetext{
55 Ibid., 26-27.

56 Ibid.

57 Ibid., 7-8.

58 Ibid.
} 